\title{
Skin cancer in solid organ transplant recipients: are mTOR inhibitors a game changer?
}

\author{
Edward K Geissler
}

\begin{abstract}
While immunosuppressive agents are necessary to prevent the rejection of transplanted organs, and are a great medical success story for protecting against early allograft loss, graft and patient survival over the long term are diminished by side effects from these same drugs. One striking long-term side effect is a high rate of skin cancer development. The skin cancers that develop in transplant recipients tend to be numerous, as well as particularly aggressive, and are therefore a major contributor to morbidity and mortality in transplant recipients. An apparent reason for the high incidence of skin cancer likely relates to suppression of immune surveillance mechanisms, but other more direct effects of certain immunosuppressive drugs are also bound to contribute to cancers of UV-exposed skin. However, over the past few years, evidence has emerged to suggest that one class of immunosuppressants, mammalian target of rapamycin (mTOR) inhibitors, could potentially inhibit skin tumour formation through a number of mechanisms that are still being studied intensively today. Therefore, in light of the high skin cancer incidence in transplant recipients, it follows that clinical trials have been conducted to determine if mTOR inhibitors can significantly reduce these post-transplant skin malignancies. Here, the problem of post-transplant skin cancer will be briefly reviewed, along with the possible mechanisms contributing to this problem, followed by an overview of the relevant clinical trial results using mTOR inhibitors.
\end{abstract}

Keywords: mTOR, Organ transplantation, Post-transplant malignancy, Calcineurin inhibitors, Skin cancer

\section{Skin cancer in transplant recipients}

Key breakthroughs in the discovery of immunosuppressive agents over the past three to four decades have expanded the routine use of solid organ transplantation to the point where demand for organs far exceed their availability. Organ transplantation has unquestionably been a success story brought about by medical research. In the current state of this success, however, the transplantation community has come to the realisation that while early organ allograft and patient survival are excellent, long-term outcomes have not improved substantially over the past couple of decades. The inadequacy of organ availability has heightened this realisation of unimproved long-term outcomes and has caused transplant organisations to search for reasons of explanation. There are indeed a number of factors responsible for poor long-term results, including chronic allograft rejection, cardiovascular disease, infectious complications and development of malignancies

Correspondence: edward.geissler@klinik.uni-regensburg.de Section of Experimental Surgery, Department of Surgery, University Hospital Regensburg, Franz-Josef-Strauss-Allee 11, Regensburg 93053, Germany
[1,2]. Regarding the latter, it is particularly difficult to accept the complication of post-transplant malignancies since these recipients typically die with a well-functioning transplant. Adding to the frustration is the fact that the malignancies commonly occur after ten or more years posttransplantation, meaning that the recipient is otherwise adapting well to the allograft over the long term by not immunologically rejecting the organ. New strategies aimed at reducing malignancies in these patients are therefore clearly needed.

By far the most common malignancies occurring in transplant recipients are skin cancers. Data from the different registries suggest that more than half of transplant recipients will experience at least one of a variety of skin malignancies [3]. The predominant epithelial skin cancer in this population is cutaneous squamous cell carcinoma (SCC), which is characterised by lesions that subsequently increase in frequency and multiplicity after the first lesion is diagnosed [4,5]; the occurrence rate is increased by approximately $65-100$-fold compared to the general population [6]. Successful strategies to combat these highly 
frequent and too often aggressive tumours would have a significant impact on the quality of life for long-term allograft recipients. Other skin tumours are also increased in organ transplant recipients, although less frequently. Basal cell carcinomas (BCC) are the second most common skin tumour post-transplantation, although they do not display a more aggressive behaviour compared to the general population $[7,8]$. Interestingly, while $\mathrm{BCC}$ are more common (4:1) than SCC in the general population, this ratio is completely reversed in transplant recipients, indicating the extraordinary predilection for SCC development in these immunosuppressed people. Highly aggressive cutaneous Merkel cell carcinomas are also increased 5-10-fold in transplant recipients [9], and Kaposi's sarcoma is dramatically increased to a rate up to 500 -fold more than age-matched controls [10]. Although otherwise rather rare, Kaposi's sarcoma accounts for approximately $6 \%$ of all malignancies in transplant recipients. Unlike SCC which tends to occur several years after transplantation, Kaposi's sarcoma occurs within the first year or two post-transplantation and develops in close association with human herpesvirus 8 (HHV-8) reactivation. Finally, it should be mentioned that cutaneous malignant melanoma shows only a slight increase in risk for transplant recipients, but high mortality rates have been reported $[11,12]$, making it a serious concern for these patients. A recent report indicates that cutaneous melanomas in kidney transplant recipients show a particularly aggressive tumour behaviour that is reflected in demonstrably poor outcomes [13].

\section{Mechanisms for increased skin cancer}

While the complex topic of causes for these various types of skin cancer cannot be comprehensively covered in this review, a few mechanisms should be mentioned for establishing a basic knowledge. The most obvious factor thought to affect cancer development in transplant recipients is systemic suppression of the immune system caused by anti-rejection drugs. There are two primary consequences of immune suppression, one being the inhibition of immune reactions capable of recognizing and destroying tumour cells [14] and the other being the permissiveness of viral infections that are associated with common skin cancers (e.g. HHV-8, Kaposi's sarcoma; human papillomavirus, SCC; Merkel cell polyomavirus, Merkel cell carcinoma) [15]. However, it is becoming increasingly clear that non-immunological factors are also important, such as direct effects of certain immunosuppressive drugs on neoplasms. For example, cyclosporine (calcineurin inhibition) is known to promote tumour cell invasiveness [16] and boost vascular endothelial growth factor-induced angiogenesis that nourishes cancer growth [17]. Importantly, cyclosporine is also known to have an inhibitory effect on DNA repair mechanisms [18], which are understandably critical for the repair of damage caused by UV light on exposed areas of the skin. Similarly, another immunosuppressant, azathioprine, is well known to be mutagenic, acting synergistically with UV radiation damage in promoting skin cancer pathogenesis [19-21]. Therefore, commonly used immunosuppressants in transplant recipients demonstrate activities that are likely to promote the development of skin tumours. Indeed, several experimental animal studies support this theory, in that treatment with daily immunosuppressive doses of cyclosporine causes tumours to grow at a faster rate $[17,22,23]$.

\section{Potential for mTOR inhibition to reduce skin cancer}

There has been much debate, testing and intensive continuing research regarding the potential anti-tumour effects of mammalian target of rapamycin (mTOR) inhibitors [24-26]. Inhibition of mTOR sets up a unique molecular scenario whereby it is plausible that various aspects of tumour development could be inhibited while at the same time causing a general immunosuppression that protects allografts from rejection. The mTOR pathway is essential for cell growth and proliferation and influences processes such as autophagy, serving as a pivotal regulatory point for the coordination of cell signalling with nutrient availability $[27,28]$. Interestingly, both cells of the immune system and of tumour entities require this type of coordination and therefore are influenced by inhibition of mTOR. Although this concise review does not allow space for going into details about the intricacies of the two different mTOR complexes, it should be mentioned here that the mTOR inhibitors used for immunosuppression in transplant recipients (sirolimus and everolimus) primarily inhibit mTOR complex $1[29,30]$, and this is where the discussion will focus; subsequently in this review, when referring to mTOR inhibition, it is generally in reference to inhibition of mTOR complex 1.

In the case of the immune system, a primary effect of mTOR inhibition is through blocking of the IL-2 proliferation signal to $\mathrm{T}$ cells, which is necessary to expand $\mathrm{T}$ cell responses directed specifically against the alloantigens expressed by the transplanted organ; thus, mTOR inhibition is highly immunosuppressive. It should also be mentioned however that mTOR inhibition has other effects on specific populations of immune cells, including inhibition of antigen-presenting cell maturation [31] and promotion of $\mathrm{T}$ regulatory cell development [32]. The complex effects of mTOR inhibition on the immune system have been reviewed elsewhere [33], but it can be summarised here that there are both inhibitory and potent stimulatory effects on immune cell populations that are likely mediated by mTOR inhibitors. Therefore, while 
clearly immunosuppressive, it should be kept in mind that mTOR inhibitors have widely different effects on immune cells, making it difficult to predict what the overall effect may be on tumour formation; indeed, the effect may vary from one tumour type to the other, depending on their immunogenicity.

Nonetheless, there is a large body of literature that indicates that mTOR inhibitors may be effective against various aspects of tumour development. Indeed, there are several possible mechanisms of action that have been described. While this is not meant to be a full review of anti-tumour mechanisms potentially involved, it is worth pointing out some of the key roles of the mTOR pathway that could impact skin cancer development. For instance, angiogenesis relies on vascular endothelial growth factor expression and signalling in supportive tumour vessel structures, which are dependent on the mTOR pathway [34]. We have shown that mTOR inhibitors dramatically block tumour angiogenesis, which leads to substantially reduced tumour growth in animal models [17]. Another aspect is the multitude of mutations in key signalling molecules that have been described upstream of the mTOR complex 1 signalling node that result in constitutive activation of the mTOR pathway and thus uncontrolled cell growth and proliferation; included in this extensive list are mutations in PTEN, TSC1/2 and Ras/Raf that lead to mTOR complex 1 activation [24]. Pkd-1 mutations can also result in triggering of the mTOR pathway and have been linked to cell proliferation and the development of polycystic kidney disease; blocking mTOR with rapamycin can substantially inhibit the proliferation of cysts in mice with this conditionally expressed mutation [35]. Therefore, inhibition of mTOR has substantial mechanistic potential to be considered overall as an anti-cancer agent.

Particularly relevant for the topic of skin cancers in transplant recipients may be the recently discovered potential anti-viral effects of mTOR inhibitors [36,37]. The close association of viral infection (as discussed earlier) with the development of different skin malignancies brings this property into focus. While the mechanisms for the anti-viral effect are not well understood yet, it seems some viruses are responsive to mTOR inhibition [37], and mTOR inhibitors have been shown to boost CD8 $\mathrm{T}$ cell responses induced by viral vaccines, even at immunosuppressive doses in non-human primates [38]. Therefore, if the anti-viral effects of mTOR inhibitors are better characterised and eventually confirmed, this may be another important mechanism to consider in the fight against post-transplant skin malignancies.

\section{Key skin cancer trials in transplant recipients}

Apart from the experimental and theoretical view towards reducing skin cancer in organ transplant recipients with mTOR inhibitors, the first clinical trials with this aim have now been published. In this part of the review, results from the key trials will be discussed (see Table 1).

\section{Australian skin cancer trial}

The first report of a randomised, multicentre clinical trial tested the effects of switching from calcineurin inhibitor-based immunosuppression to sirolimus on the risk for development of non-melanoma skin cancer (NMSC) in renal transplant recipients [39]. A total of 87 transplant patients at a high risk for NMSC were randomised at least 1 year after transplantation to continue on a calcineurin inhibitor-based immunosuppression or be switched to sirolimus; the primary endpoint was number of biopsy-confirmed new NMSC per patient per year. Over a 2-year observation period, SCC occurred at a significantly lower rate in the sirolimus group of patients, although the rate of basal cell carcinomas was the same. Also, a lower rate of new NMSC developed in the sirolimus-converted group. Furthermore, it took more than twofold the number of days for a new NMSC to occur in the sirolimus versus control group of patients. Importantly, the conversion to sirolimus did not result in an increased risk for having an acute kidney rejection episode, but there was a high rate of treatment discontinuation in the sirolimus group (42.6\%) due to typical well-known side effects associated with mTOR inhibitor

Table 1 Randomised multicentre clinical trials with skin cancer as the primary endpoint

\begin{tabular}{lllll}
\hline Study & Patient number & Primary endpoint & Primary endpoint result & Comments \\
\hline Australian study & Control: $47(23)^{a}$ & Number of new NMSC/patient/year & Significant & A significant decrease in SCC, but not BCC \\
mTORi: $39(19)$ & & Significant & $\begin{array}{l}\text { patients with 1 prior SCC benefited significantly; } \\
\text { those with multiple previous SCC did not } \\
\text { significantly benefit }\end{array}$ \\
& Control: $56(12)$ & Survival free of new SCC & & HR improved in 1-year analysis, but did not after \\
RESCUE & mTORi: $64(22)$ & & Not significant & 2-year follow-up
\end{tabular}

SCC squamous cell carcinoma, BCC basal cell carcinoma, NMSC non-melanoma skin cancer, Control patients not receiving mTOR inhibitors, $m$ TORi mTOR inhibitor group, $H R$ hazard ratio.

${ }^{a}$ Number in parentheses represents patients dropping out of study. For the definition of a dropout and number of patients that completed the study according to the protocol, see the individual publications. 
use. Nonetheless, the switch from calcineurin inhibitor to mTOR inhibitor did have a positive clinically significant effect on skin cancer development in this study.

\section{TUMORAPA}

Within a couple of months after the release of the Australian trial results, Euvrard and colleagues published data from a similar clinical trial that focussed on SCC in kidney transplant recipients [40]. This trial used a combination of data from two registered trials, TUMORAPA-1 and TUMORAPA-N, which aimed to enrol patients with a first SCC and after multiple SCC post-transplantation, respectively. A total of 120 patients were enrolled in this combined study, which was a calcineurin inhibitor to sirolimus conversion protocol looking at SCC-free survival 2 years after randomisation. The rate of SCC-free survival was significantly longer with sirolimus conversion, where $22 \%$ of patients developed new SCC compared to $39 \%$ in the group maintained on calcineurin inhibitors. An important observation in this study was that significance in the mTOR inhibitor effect held in the group of patients that had only a single pre-randomisation SCC, but was lost when recipients had multiple SCC prior to entry into the study; it should be added however that the study may not have been adequately powered to see such a difference. Another potentially important observation was that patients did experience significant side effects causing treatment discontinuation in the sirolimus conversion group, but these side effects were substantially fewer when a slower conversion (over $>7$ days) was performed. The Australian and TUMORAPA trials in large part reached a similar conclusion that mTOR inhibitors inhibit skin cancer in high-risk kidney transplant recipients.

\section{RESCUE}

Another related clinical trial in the Netherlands and the United Kingdom was performed during approximately the same time frame as the previously cited trials and is referred to as the RESCUE trial [41]. Similar to the first two trials discussed, RESCUE was a randomised, multicentre study with a 2-year follow-up in kidney transplant recipients that had experienced at least one prerandomisation SCC. A total of 155 patients were randomised into a group maintained on their non-mTOR inhibitor-based regime or into an arm where conversion to sirolimus was performed. The primary endpoint was met when the patient developed a new SCC within the 2 -year observation period. Results from this trial are interesting to contrast with the other two trials, since no significant decrease in new SCC (the primary endpoint) was observed in the sirolimus group as a whole after 2 years, but additional analyses revealed that there was a significant $50 \%$ decrease in risk (measured by hazard ratio) after only 1 year of observation. A provocative conclusion to this finding is that mTOR inhibitors delay, but do not prevent, skin cancers in these patients; a longer-term follow-up would be necessary to begin to address this issue. An additional finding in this study was a substantial improvement in hazard ratio for those patients that entered the trial with only one previous SCC, which is consistent with the results from TUMORAPA. As with the other trials, a high discontinuation rate (39\%) was observed in the sirolimus group due to adverse side effects.

\section{Additional useful trial results}

It should be mentioned that other trials have investigated the effect of mTOR inhibitors on development of skin cancer in transplant recipients, including a small randomised single-centre German study published by Salgo and colleagues [42]. This trial showed also that sirolimus conversion in renal transplant recipients has an inhibitory effect on development of NMSC, where only 1/16 sirolimus-converted versus 8/17 control group patients developed skin cancer. Results from large sirolimus conversion trials, where de novo malignancies were examined only as secondary endpoints, also show a beneficial effect in terms of skin cancer occurrence [43,44]. Evidence is emerging to show that everolimus is also likely to have a similar inhibitory effect on skin cancer in transplant recipients $[45,46]$. Finally, it is important to point out the success reported in the treatment of Kaposi's sarcoma with mTOR inhibitor conversion in transplant recipients. Several key studies have shown a dramatic effect of mTOR inhibitor conversion on lesion stabilisation, reduction and even disappearance [47-49]. Kaposi's sarcoma is potentially a good target for mTOR inhibitors, since they are highly vascular tumours and linked directly with HHV-8 viral infection.

\section{Conclusions}

Skin cancer is a substantial problem in organ transplant recipients that is attributed to the immunosuppressive drugs that are most commonly used to prevent allograft rejection. In particular, evidence suggests that the calcineurin inhibitors and azathioprine contribute to the development of skin neoplasms both through paralysis of immune surveillance and by promotion of tumour vascularisation, cancer cell invasiveness and exacerbation of DNA damage or inhibition of DNA repair. While suppression of the immune reactivity against cancer cannot be completely avoided with an immunosuppressive drug, mTOR inhibitors have a unique potential to both suppress an immune response to the organ allograft and promote mechanisms that can potentially inhibit tumour development. The basic research to better understand this phenomenon continues to evolve at a steady pace, but in the meantime, clinical trials in transplant recipients already indicate that skin cancer can be reduced by 
substitution of calcineurin inhibitors with mTOR inhibitors. Results from clinical studies suggest also that patients at a high risk for skin cancer receive the greatest benefit when mTOR inhibitors are used early, before multiple lesions have already developed. The key to taking advantage of this benefit will be the institution of improved protocols that minimise the adverse side effects of mTOR inhibitor use in transplant recipients that undergo immunosuppression switching. Therefore, mTOR inhibitors could be a "game changer" to reduce the problem of post-transplantation skin cancer, but more research will be necessary to optimise clinical protocols that will be widely acceptable for high-risk patients that can benefit from this general strategy.

\section{Competing interests}

The author declares that he has no competing interests.

\section{Declarations}

This article is based on a presentation from the 13th International Symposium on Transplantation held on 22-23 April 2013, in Rome, Italy. Additional articles from the symposium are to be published as Transplantation Research Volume 4 Suppl 1, 2015 (http://www. transplantationresearch.com/supplements/4/S1) subject to acceptance following peer review. The symposium and publication based on these proceedings have been supported by an unrestricted educational grant from Pfizer Inc., who had no influence on the topics featured, authors or editorial content of the publications in Transplantation Research. Editorial support was provided by Synergy Medical, Surrey, UK and was funded by Pfizer Inc. The articles have been through the journal's standard peer review process.

Received: 24 November 2014 Accepted: 16 December 2014

Published online: 14 January 2015

\section{References}

1. Penn I. Occurrence of cancers in immunosuppressed organ transplant recipients. Clin Transplant. 1998;147-58

2. Briggs JD. Causes of death after renal transplantation. Nephrol Dial Transplant. 2001;16:1545-9.

3. Euvrard S, Kanitakis J, Claudy A. Skin cancers after organ transplantation. N Engl J Med. 2003;348:1681-91.

4. Euvrard S, Kanitakis J, Decullier E, Butnaru AC, Lefrancois N, Boissonnat P, et al. Subsequent skin cancers in kidney and heart transplant recipients after the first squamous cell carcinoma. Transplantation. 2006:81:1093-100.

5. Wisgerhof HC, Edelbroek JR, de Fijter JW, Haasnoot GW, Claas FH, Willemze R, et al. Subsequent squamous- and basal-cell carcinomas in kidney-transplant recipients after the first skin cancer: cumulative incidence and risk factors. Transplantation. 2010;89:1231-8.

6. Lindelöf B, Sigurgeirsson B, Gäbel H, Stern RS. Incidence of skin cancer in 5356 patients following organ transplantation. Br J Dermatol. 2000;143:513-9.

7. Mertz KD, Proske D, Kettelhack N, Kegel C, Keusch G, Schwarz A, et al. Basal cell carcinoma in a series of renal transplant recipients: epidemiology and clinicopathologic features. Int J Dermatol. 2010;49:385-9.

8. Kanitakis J, Alhaj-lbrahim L, Euvrard S, Claudy A. Basal cell carcinomas developing in solid organ transplant recipients: clinicopathologic study of 176 cases. Arch Dermatol. 2003;139:1133-7.

9. Buell JF, Trofe J, Hanaway MJ, Beebe TM, Gross TG, Alloway RR, et al. Immunosuppression and Merkel cell cancer. Transplant Proc. 2002;34:1780-1.

10. Penn I. Cancers in renal transplant recipients. Adv Ren Replace Ther. 2000;7:147-56.

11. Hollenbeak CS, Todd MM, Billingsley EM, Harper G, Dyer AM, Lengerich EJ. Increased incidence of melanoma in renal transplantation recipients. Cancer. 2005; 104:1962-7.

12. Le Mire L, Hollowood K, Gray D, Bordea C, Wojnarowska F. Melanomas in renal transplant recipients. Br J Dermatol. 2006;154:472-7.
13. Vajdic CM, Chong AH, Kelly PJ, Meagher NS, van Leeuwen MT, Grulich AE, et al. Survival after cutaneous melanoma in kidney transplant recipients: a population-based matched cohort study. Am J Transplant. 2014;14:1368-75.

14. Rovira J, Sabet-Baktach M, Eggenhofer E, Lantow M, Koehl GE, Schlitt HJ, et al. A color-coded reporter model to study the effect of immunosuppressants on CD8 ${ }^{+}$T-cell memory in antitumor and alloimmune responses. Transplantation. 2013;95:54-62.

15. Nindl I, Rosl F. Molecular concepts of virus infections causing skin cancer in organ transplant recipients. Am J Transplant. 2008;8:2199-204.

16. Hojo M, Morimoto T, Maluccio M, Asano T, Morimoto K, Lagman M, et al. Cyclosporine induces cancer progression by a cell-autonomous mechanism. Nature. 1999;397:530-4.

17. Guba M, von Breitenbuch P, Steinbauer M, Koehl G, Flegel S, Hornung M, et al. Rapamycin inhibits primary and metastatic tumor growth by antiangiogenesis: involvement of vascular endothelial growth factor. Nat Med. 2002;8:128-35.

18. Herman M, Weinstein T, Korzets A, Chagnac A, Ori Y, Zevin D, et al. Effect of cyclosporin A on DNA repair and cancer incidence in kidney transplant recipients. J Lab Clin Med. 2001;137:14-20.

19. de Gruijl FR, van Kranen HJ, Mullenders LH. UV-induced DNA damage, repair, mutations and oncogenic pathways in skin cancer. J Photochem Photobiol B. 2001;63:19-27.

20. O'Donovan P, Perrett CM, Zhang X, Montaner B, Xu YZ, Harwood CA, et al. Azathioprine and UVA light generate mutagenic oxidative DNA damage. Science. 2005;309:1871-4.

21. Hofbauer GF, Attard NR, Harwood CA, McGregor JM, Dziunycz P, lotzova-Weiss G, et al. Reversal of UVA skin photosensitivity and DNA damage in kidney transplant recipients by replacing azathioprine. Am J Transplant. 2012;12:218-25.

22. Koehl G, Andrassy J, Guba M, Richter S, Kroemer A, Scherer MN, et al. Rapamycin protects allografts from rejection while simultaneously attacking tumors in immunosuppressed mice. Transplantation. 2004;77:1319-26.

23. Duncan FJ, Wulff BC, Tober KL, Ferketich AK, Martin J, Thomas-Ahner JM, et al. Clinically relevant immunosuppressants influence UVB-induced tumor size through effects on inflammation and angiogenesis. Am J Transplant. 2007;7:2693-703.

24. Geissler EK, Schlitt HJ, Thomas G. mTOR, cancer and transplantation. Am J Transplant. 2008;8:2212-8.

25. Bjornsti MA, Houghton PJ. The tor pathway: a target for cancer therapy. Nat Rev Cancer. 2004;4:335-48.

26. Guertin DA, Sabatini DM. Defining the role of mTOR in cancer. Cancer Cell. 2007;12:9-22.

27. Fingar DC, Blenis J. Target of rapamycin (TOR): an integrator of nutrient and growth factor signals and coordinator of cell growth and cell cycle progression. Oncogene. 2004;23:3151-71.

28. Jacinto E, Hall MN. Tor signalling in bugs, brain and brawn. Nat Rev Mol Cell Biol. 2003;4:117-26.

29. Sehgal SN. Sirolimus: its discovery, biological properties, and mechanism of action. Transplant Proceedings. 2003;35:7S-14.

30. Webster AC, Lee WW, Chapman JR, Craig JC. Target of rapamycin inhibitors (sirolimus and everolimus) for primary immunosuppression of kidney transplant recipients: a systematic review and meta-analysis of randomized trials. Transplantation. 2006;81:1234-48.

31. Turnquist HR, Raimondi G, Zahorchak AF, Fischer RT, Wang Z, Thomson AW. Rapamycin-conditioned dendritic cells are poor stimulators of allogeneic $\mathrm{CD}^{+} \mathrm{T}$ cells, but enrich for antigen-specific Foxp3 ${ }^{+} \mathrm{T}$ regulatory cells and promote organ transplant tolerance. J Immunol. 2007;178:7018-31.

32. Battaglia M, Stabilini A, Roncarolo MG. Rapamycin selectively expands CD4 + CD25 + FoxP3+ regulatory T cells. Blood. 2005;105:4743-8.

33. Geissler EK. The influence of mTOR inhibitors on immunity and the relationship to post-transplant malignancy. Transplant Res. 2013;2:S2.

34. Phung TL, Ziv K, Dabydeen D, Eyiah-Mensah G, Riveros M, Perruzzi C, et al. Pathological angiogenesis is induced by sustained Akt signaling and inhibited by rapamycin. Cancer Cell. 2006;10:159-70.

35. Novalic Z, van der Wal AM, Leonhard WN, Koehl G, Breuning MH, Geissler EK et al. Dose-dependent effects of sirolimus on mTOR signaling and polycystic kidney disease. J Am Soc Nephrol. 2012;23:842-53.

36. Egli A, Köhli S, Dickenmann M, Hirsch HH. Inhibition of polyomavirus BK-specific T-cell responses by immunosuppressive drugs. Transplantation. 2009;88:1161-8.

37. Brennan DC, Aguado JM, Potena L, Jardine AG, Legendre C, Säemann MD, et al. Effect of maintenance immunosuppressive drugs on virus 
pathobiology: evidence and potential mechanisms. Rev Med Virol. 2013:23:97-125.

38. Turner AP, Shaffer VO, Araki K, Martens C, Turner PL, Gangappa S, et al. Sirolimus enhances the magnitude and quality of viral-specific $\mathrm{CD} 8^{+} \mathrm{T}$-cell responses to vaccinia virus vaccination in rhesus macaques. Am J Transplant. 2011:11:613-8.

39. Campbell SB, Walker R, Tai SS, Jiang Q, Russ GR. Randomized controlled trial of sirolimus for renal transplant recipients at high risk for nonmelanoma skin cancer. Am J Transplant. 2012;12:1146-56.

40. Euvrard S, Morelon E, Rostaing L, Goffin E, Brocard A, Tromme I, et al. Sirolimus and secondary skin-cancer prevention in kidney transplantation. N Engl J Med. 2012;367:329-39.

41. den Akker JMH-v, Harden PN, Hoitsma AJ, Proby CM, Wolterbeek R, Bouwes Bavinck JN, et al. Two-year randomized controlled prospective trial converting treatment of stable renal transplant recipients with cutaneous invasive squamous cell carcinomas to sirolimus. J Clin Oncol. 2013;31:1317-23.

42. Salgo R, Gossmann J, Schöfer H, Kachel HG, Kuck J, Geiger H, et al. Switch to a sirolimus-based immunosuppression in long-term renal transplant recipients: reduced rate of (pre-)malignancies and nonmelanoma skin cancer in a prospective, randomized, assessor-blinded, controlled clinical trial. Am J Transplant. 2010;10:1-9.

43. Campistol JM, Eris J, Oberbauer R, Friend P, Hutchison B, Morales JM, et al. Sirolimus therapy after early cyclosporine withdrawal reduces the risk for cancer in adult renal transplantation. J Am Soc Nephrol. 2006;17:581-9.

44. Schena FP, Pascoe MD, Alberu J, del Carmen RM, Oberbauer R, Brennan DC, et al. Conversion from calcineurin inhibitors to sirolimus maintenance therapy in renal allograft recipients: 24-month efficacy and safety results from the CONVERT trial. Transplantation. 2009;87:233-42.

45. Euvrard S, Boissonnat P, Roussoulieres A, Kanitakis J, Decullier E, Claudy A, et al. Effect of everolimus on skin cancers in calcineurin inhihitor-treated heart transplant recipients. Transpl Int. 2010;23:855-7.

46. Alter M, Satzger I, Schrem H, Kaltenborn A, Kapp A, Gutzmer R. Non-melanoma skin cancer is reduced after switch of immunosuppression to mTOR-inhibitors in organ transplant recipients. J Dtsch Dermatol Ges. 2014;12:480-8.

47. Stallone G, Schena A, Infante B, Di Paolo S, Loverre A, Maggio G, et al. Sirolimus for Kaposi's sarcoma in renal-transplant recipients. N Engl J Med. 2005:352:1317-23.

48. Campistol JM, Gutierrez-Dalmau A, Torregrosa JV. Conversion to sirolimus: a successful treatment for posttransplantation Kaposi's sarcoma. Transplantation. 2004;77:760-2.

49. Lebbé $C$, Legendre $C$, Francès $C$. Kaposi sarcoma in transplantation. Transplant Rev. 2008;22:252-61.

\section{Submit your next manuscript to BioMed Central and take full advantage of:}

- Convenient online submission

- Thorough peer review

- No space constraints or color figure charges

- Immediate publication on acceptance

- Inclusion in PubMed, CAS, Scopus and Google Scholar

- Research which is freely available for redistribution 